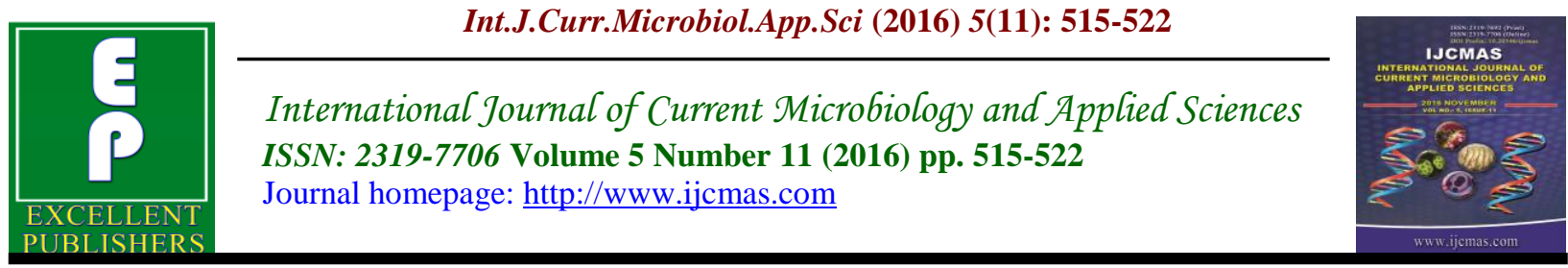

Original Research Article

http://dx.doi.org/10.20546/ijcmas.2016.511.060

\title{
Prevalence of ESBL and MBL producing Acinetobacter Isolates in Clinical Specimens in Tertiary Care Hospital, Assam, India
}

\author{
Harekrishna Nath* and Dipa Barkataki \\ Department of Microbiology, Gauhati Medical College and Hospital, Assam, India \\ *Corresponding author
}

Keywords

MDR

A.baumannii, ESBL producer, MBL producer, Matelo Beta Lactamese

Article Info

Accepted:

23 October 2016

Available Online:

10 November 2016

\section{A B S T R A C T}

Outbreaks of infection caused by strains of A. baumannii resistant to multiple antibiotic classes, including carbapenems, are a serious concern in many specialized hospital units, including intensive care units (ICUs) Clinical isolates of Acinetobacter species initially retained at least partial susceptibility against the 3rd and 4th generations viz cephalosporins, fluoroquinolones, semisynthetic aminoglycosides, carbapenems and $100 \%$ susceptibility to imipenem but the clinical utility of this class of antimicrobial is increasingly being jeopardized by the emergence of both enzymatic and membrane-based mechanisms of resistance. The increase in the number of MBLs in A. baumannii is an ominous development in the global emergence of resistance to $\beta$-lactams. Keeping this things mind study was carried out to know the prevalence of ESBL \& MBL producing Acinetobacter isolates in tertiary care hospital. A total of 31 no's Acinetobacter isolation was done from clinical specimens from different medical wards, surgical wards and ICU department of GMCH for a period of one year from August 2014--July 2015. Isolated Acinetobacter spp were tested for ESBL (Extended spectrum Beta Lactamase) by double disc synergy and MBL (Matelo Beta Lactamese) by imipenem and Imepenem+EDTA E-test method. Out of the total of 31 Acinetobacter isolates, MDR isolates found to be 13(41.94\%) of which A.baumannii 12(92.30\%) and Acinetobacter lwoffii 1(7.69\%). MDR Acinetobacter were sensitive to Polymixin B (100\%) followed by Tigecycline, colistin $(92.85 \%)$. Out of the total of 31 Acinetobacter isolates, $32.25 \%$ isolates are ESBL producer and $38.7 \%$ of isolates were MBL producer and all MBL producers were isolated from ICU. Maximum MDR, ESBL and MBL isolation rates were seen from tracheal aspirates. In our study $32.25 \%$ isolates and $38.7 \%$ of isolates were ESBL and MBL producer respectively and all MBL producers were isolated from ICU. Therefore early detection is necessary to screen and confirm beta lactamase mediated resistant strains to avoid treatment failure and prevent the spread of MDR.

\section{Introduction}

Acinetobacter spp play a significant role in the colonization and infection of patients admitted to hospitals (Bergogne-Berezin et al., 1996). Acinetobacter is a Gram Negative
Coccobacillus (Munoz-Price et al., 2008; Schreckenberger et al., 2007). The organism is ubiquitous in nature; its ability to survive in varying temperatures, $\mathrm{pH}$ conditions, and 
on dry, moist surfaces helps in the transmission and propagation of this organism in the hospital setting (Health care associated infection; Manchanda et al., 2010).

It typically colonizes skin, the respiratory, urinary, gastrointestinal tract wounds and indwelling plastic devices of the hospitalized patients can cause infections in burn, trauma, mechanically ventilated and immunocompromised patients. It shows a special predilection for the ICU (Patwardhan et al., 2008).

Clinical isolates of Acinetobacter species initially retained at least partial susceptibility against the 3rd and 4th generations viz cephalosporins, fluoroquinolones, semisynthetic aminoglycosides, carbapenems and 100\% susceptibility to imipenem (Montefour et al., 2008). In addition, intrinsic resistance and multi-drug resistance (MDR) pose a global medical challenge (Li et al., 2007). Carbapenems which were once the mainstay of therapy are no longer effective in controlling the infections caused by this organism. Although these drugs are still active against the vast majority of $A$. baumannii strains worldwide, the clinical utility of this class of antimicrobial is increasingly being jeopardized by the emergence of both enzymatic and membrane-based mechanisms of resistance. The increase in the number of MBLs in $A$. baumannii is an ominous development in the global emergence of resistance to $\beta$-lactams. Rasmussen and Bush (Rasmussen et al., 1997) reported, carbapenem resistance can be due to acquired carbapenemase production. Carbapenem resistance is mainly due to either reduced levels of drug accumulation or increased expression levels of the pump efflux. Other risk factors responsible for colonization and infection with MBL producers include age of patient, duration of hospitalization, underlying diseases like diabetes, tumours or overcrowding in the hospital wards. Heritier et al., (2005) reported that Acinetobacters may develop resistance to carbapenems through various combined mechanisms, including AmpC stable depression, decreased permeability, altered penicillinbinding proteins (PBPs) and, rarely, efflux pump over expression.

Outbreaks of infection caused by strains of A. baumannii resistant to multiple antibiotic classes, including carbapenems, are a serious concern in many specialized hospital units, including intensive care units (ICUs) (David et al., 2012). The foremost implication of infection with carbapenemresistant $A$. baumannii is the need to use "last-line" antibiotics such as colistin, polymyxin B, or Tigecycline.

The main objectives of this study prevalence of ESBL \& MBL producing Acinetobacter isolates in tertiary care hospital.

\section{Materials and Methods}

A total of 31 no's Acinetobacter isolation was done from clinical specimens endotracheal aspirate and bronchoalveolar lavage, Blood /catheter tips, Pus / wound swab, Body fluids (Pleural fluid, ascetic fluid and cerebrospinal fluid etc) collected from different medical wards, surgical wards and ICU department of GMCH for a period of one year from August 2014--July 2015.

Samples were collected from patients of all age groups. The study was commenced with ethical approval and clearance certificate from the IEC, GMCH. Species identification was done by KBO14 and by Vitek2 compact. Antimicrobial susceptibility of isolated Acinetobacter spp were tested by modified Kirby-Bauer disc diffusion method 
as per the recommendation of Clinical and Laboratory Standard Institute (CLSI)and VITEK 2 compact AST 281) as per guideline.

Acinetobacter baumannii ATCC19606 and Acinetobacter lwoffii ATCC 15309 were used as quality control strains. VITEK 2 compact -identification and sensitivity testing confirmation done by (GN card and AST 281) as per guideline.

The isolates were tested against antibiotics Table 1. Isolates showing resistance to at least three categories of drugs i.e. penicillins and cephalosporins, fluroquinolones, and aminoglycosides, were considered multidrug resistant (Abbo et al., 2005). Extensive drug resistant (XDR) Acinetobacter were isolates displaying resistance to carbapenems in addition to resistance to penicillins and cephalosporins, fluroquinolones, and aminoglycosides. Pan resistant Acinetobacter spp. was defined as Acinetobacter isolate that is resistant to the whole panel of antibiotics (Manchanda et al., 2010).

Isolated Acinetobacter spp were tested for ESBL (Extended spectrum Beta Lactamase) by double disc synergy and MBL (Matelo Beta Lactamese) by imipenem and Imepenem+EDTA E-test method.

\section{Phenotypic detection of ESBL}

Ceftazidime-resistant isolates were screened for producing ESBL. The double disk method was used for detection of this enzyme. Then the suspension was streaked onto Mueller-Hinton agar plates (Hi Media, Mumbai, India). A disc of either ceftazidime $(30 \mu \mathrm{g})$ or cefotaxime alone $(30 \mu \mathrm{g})$ in combination with clavulanic acid $(30 \mu \mathrm{g} / 10$ $\mu \mathrm{g})$ was placed at the distance of 20 $\mathrm{mm}$ (centre to centre). After incubation overnight at $35^{\circ} \mathrm{C}$, a positive test result was considered as a $5 \mathrm{~mm}$ increase in inhibition halo compared with a disk without clavulanic acid.

\section{Phenotypic detection of MBL}

Phenotypic detection of MBL Imipenemresistant isolates were screened for producing MBL. The double disk method was used to detect this enzyme.

Imipenem-resistant isolates were screened for producing MBL. Merpenem(MRP) with or without EDTA Ezy MIC ${ }^{\text {TM }}$ strip (EM 092) method was used to detect this enzyme. Colonies from overnight cultures on blood agar plates were suspended in peptone water and adjusted $0.5 \mathrm{McF}$ arland standard. Then the suspension was streaked onto MuellerHinton agar plates (HiMedia, Mumbai, India).

After incubation overnight at $35^{\circ} \mathrm{C}$, a positive test result was considered as a $\mathrm{MRP} / \mathrm{MRP}+\mathrm{EDTA}>=8 ; \mathrm{MBL}$ positive strain.

\section{Results and Discussion}

Out of the total of 31 Acinetobacter isolates, Acinetobacter baumannii was the predominant species $28(90.32 \%)$ isolated, followed by Acinetobacter lwoffii 2 (6.45\%) and Acinetobacter hemolyticus1(3.23\%). In the present study, the highest number of isolates $32.26 \%$ (10) in age group 21-40 years and $51.61 \%$ (16) Acinetobacter spp isolated in male. Acinetobacter species isolated from patients in ICU was $80.65 \%$ (25) and other Non ICU hospitalized patients showed lower isolation rates $19.35 \%$. Out of the total of 31 Acinetobacter isolates, MDR isolates found to be $13(41.94 \%)$ of which A.baumannii 12(92.30\%) and Acinetobacter lwoffii 1 (7.69\%). MDR Acinetobacter were sensitive to Polymixin B (100\%) followed by 
Tigecycline, colistin (92.85\%) Minocycline and cefoperazone sulbactum (Table-1).

Out of the total of 28 A.baumannii 13 $(46.43 \%)$ found to be ceftazidime and cefipime Resistant and Carbapenem resistance found in $42.85 \%$ A.baumannii. Out of the total of 31 Acinetobacter isolates, $32.25 \%(10)$ isolates are ESBL producer and $38.7 \%$ (12) of isolates were MBL producer and all MBL producers were isolated from ICU.

In the present study, out of 31 Acinetobacter spp, the predominant species was $A$. baumannii (90.32\%) followed by A.lwoffii (6.45\%) and A.hemolyticus (3.23\%). Similarly, Rahul Kamble et al., (2012) isolated (87.2\%) A.baumannii followed by A. haemolyticus $(9.3 \%)$ and A. lwoffii (3.5\%). Similarly by Sinha et al., reported Acinetobacter baumannii as the predominant species $(92.14 \%)$, while Acinetobacter lwoffii (6.42\%) and
Acinetobacter haemolyticus (1.42\%). Kalidas Rit et al., 2010; Major infections found in different medical wards, surgical wards and ICU were due to Acinetobacter baumannii (74.02\%), A. lowfii (14.2\%), A. haemolyticus (7.79\%), A. junii (3.8\%) among Acinetobacter species. Predominance A.calcoaceticus baumannii complex is perhaps its non fastidious natures and higher prevalence in hospital environment. Acinetobacter spp. have emerged as particularly important organisms in intensive care units (ICUs), and this is probably related, at least in part, to the increasingly invasive diagnostic and therapeutic procedures used in hospital ICUs in recent years.

In our study, Acinetobacter species isolated from patients in ICU most frequently $(80.65 \%)$ and A.baumannii was the predominant species similar to study done by Sharma et al., (2015) and Muntasir I. Omer et al., (2014).

Table.1 Antimicrobial susceptibility of A.baumannii isolated from infected patients

\begin{tabular}{|l|c|c|}
\hline Antimicrobial Agent & Breakpoint(micr/ml)MIC & Resistant \% (A.baumannii $\mathrm{n}=28)$ \\
\hline Amikacin & $\mathrm{s}<=16 \mathrm{R}>=64$ & 42.85 \\
\hline Gentamycin & $\mathrm{s}<=4 \mathrm{R}>=16$ & 42.85 \\
\hline Aztreonam & $\mathrm{s}<=8 \mathrm{R}>=32$ & 60.71 \\
\hline Ceftazidime & $\mathrm{s}<=8 \mathrm{R}>=32$ & 46.43 \\
\hline Cefepime & $\mathrm{s}<=8 \mathrm{R}>=32$ & 46.43 \\
\hline Cefoperazone+Sulbactum & $\mathrm{s}<=32 \mathrm{R}>=64$ & 14.29 \\
\hline Doripenum & $\mathrm{s}<=2 \mathrm{R}>=8$ & 42.85 \\
\hline Imipenem & $\mathrm{s}<=4 \mathrm{R}>=16$ & 42.85 \\
\hline Meropenem & $\mathrm{s}<=4 \mathrm{R}>=16$ & 42.85 \\
\hline Ciprofloxacin & $\mathrm{s}<=1 \mathrm{R}>=4$ & 50 \\
\hline Levofloxacin & $\mathrm{s}<=2 \mathrm{R}>=8$ & 46.43 \\
\hline Minocycline & $\mathrm{s}<=8 \mathrm{R}>=16$ & 14.29 \\
\hline Tigecycline & $\mathrm{s}<=4 \mathrm{R}>=8$ & 3.57 \\
\hline Colistin & $\mathrm{s}<=4 \mathrm{R}>=16$ & 7.14 \\
\hline Cotrimoxazole & $\mathrm{s}<=20 \mathrm{R}>=320$ & 42.85 \\
\hline Polymixin B & $\mathrm{s}<=2 \mathrm{R}>=4$ & 0 \\
\hline
\end{tabular}


Table.2 Sample wise MDR Acinetobacter distribution

\begin{tabular}{|l|l|l|}
\hline \multirow{2}{*}{ Origin of sample } & \multicolumn{2}{|c|}{ Acinetobacter spp } \\
\cline { 2 - 3 } & Total $\mathrm{n}=31$ & MDR $\mathrm{n}=13$ \\
\hline Respiratory & 14 & $11(84.61 \%)$ \\
\hline Wound swab & 5 & $2(15.38 \%)$ \\
\hline Blood & 4 & 0 \\
\hline Urine & 6 & 0 \\
\hline Body fluid & 2 & 0 \\
\hline
\end{tabular}

Fig.1 Distribution of MDR Acinetobacter in ICU and INDOOR

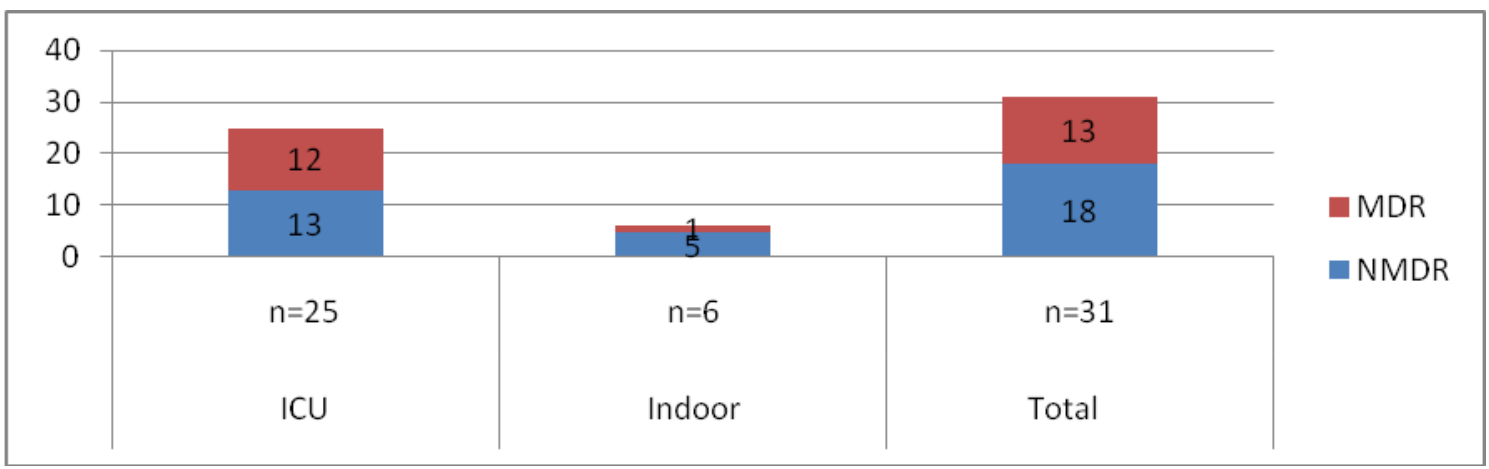

Fig.2 Prevalence of ESBL and MBL Acinetobacter

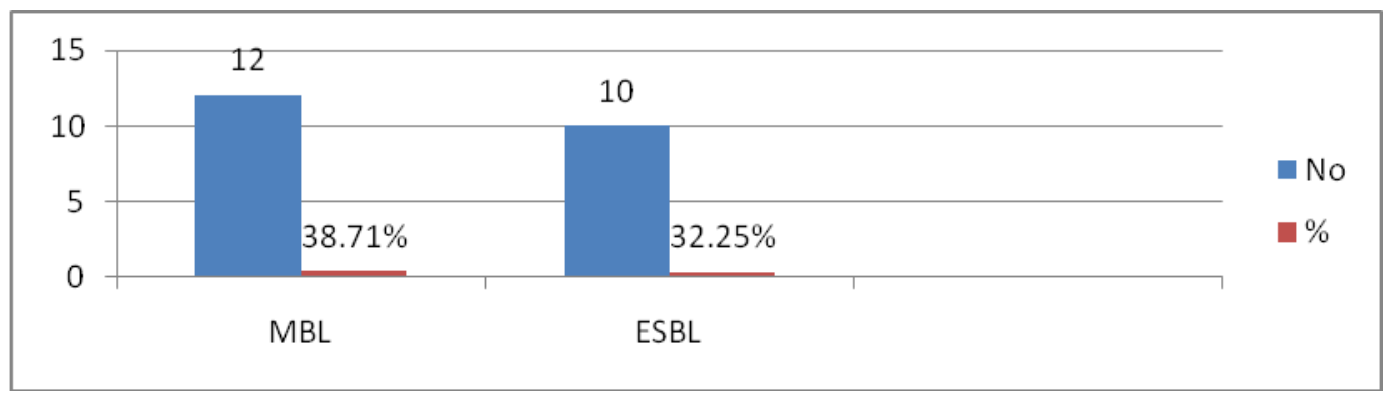

Fig.3 Distribution of ESBL and MBL Acinetobacter spp

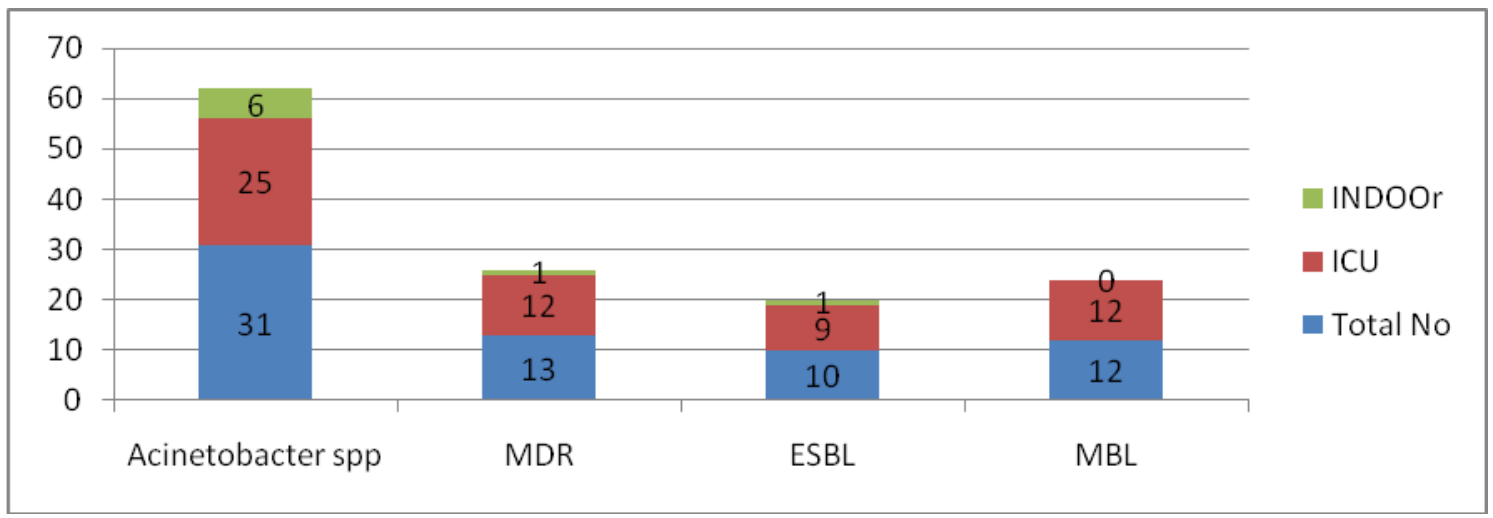


This result corroborates the fact that a lot of risk factors associated with Acinetobacter infection exist in the ICU like potential environmental reservoirs for A.baumannii, opportunities for cross transmission, sick, immunocompromised patients who are colonized, patients having multiple wounds and indwelling devices, heavy use of broad spectrum antibiotics and frequent contamination of the hands of health care workers while patient care.

In the present study, Acinetobacter isolates, $32.25 \%(10)$ isolates are ESBL producer and $38.7 \%$ (12) of isolates were MBL producer. Similarly ESBL production was detected in 28 per cent of the isolates in a study done by Sinha et al., In a study done at AIIMS, New Delhi, $48.72 \%$ of A.baumannii strains were ascertained to be MBL- enzyme producers by the same method, thus implying rapid spread of resistance amongst this pathogen. In another study done by Neetu Gupta et al., (2015), $31.5 \%$ of Acinetobacter species were ESBL producer by the DDST and $14.4 \%$ were MBL producers by the combined disc diffusion test.

Maximum MDR, ESBL and MBL isolation rates were seen from tracheal aspirates followed by wound swabs. High isolation of Acinetobacters in respiratory samples is perhaps due to its ability to survive in varying temperatures, $\mathrm{pH}$ conditions, and on dry, moist surfaces which helps in the transmission and propagation of this organism in the hospital setting.

In conclusion, A.baumannii is becoming a global medical challenge due to the emergence of multi-drug resistance. In our study $32.25 \%$ isolates and $38.7 \%$ of isolates were ESBL and MBL producer respectively and all MBL producers were isolated from ICU. $\beta$-lactamase mediated resistance mechanisms are accounting very high in the multidrug resistant isolates of Acinetobacter species. Therefore early detection is necessary to screen and confirm beta lactamase mediated resistant strains to avoid treatment failure and prevent the spread of MDR. Thus, due to such high prevalence of resistance, antibiotics must be used judiciously by the clinicians and appropriate infection control measures need to be implemented to control the spread of infections in hospitals.

\section{Acknowledgement}

We owe our great thanks to DBT, Tezpur for financial support in our study.

\section{References}

Abbo, A., Venezia, S.N., Muntz, Z.H., Krichali, T., Igra, Y.S., Carmeli, Y. $2005 . \quad$ Multidrugresistant Acinetobacterbaumannii . Em erg. Infect. Dis., 11(1): 22-9. [PMC free article] [PubMed].

Bergogne-Berezin, E., Towner, K.J. 1996. Acinetobacter spp. As nosocomial pathogens: microbiological, clinical and epidemiological features. Clin. Microbiol. Rev., 9: 148-65.

David, L., Paterson, Samuel, I., Miller, 2012. Chapter 150, In: Harrison's Principles of Internal Medicine, $18^{\text {th }}$ Edition, (Mc Graw Hill) Editors: Dan longo, Dennis Kasper, J. Larry Jameson, Anthony Fauci, Stephen Hauser, Joseph Loscalzo, Vol.1, pg 1258-1260.

Goel, V., Hogade, S.A., Karadesai, S.G. 2013. Prevalence of extendedspectrum beta-lactamases, AmpC beta-lactamase, and metallo-betalactamase producing Pseudomonas aeruginosa and Acinetobacter baumannii in an intensive care unit in 
a tertiary care hospital. J. Sci. Soc., 40: 28- 31.

Health care associated infectionsAcinetobacter in health care settings. CDC 2010. Available from: http://www.cdc.gov/HAI/organisms/A cinetobacter.html.

Heritier, C., Poirel, L., Lambert, T., Nordmann, P. 2005. Contribution of acquired carbapenem-hydrolyzing oxacillinases to carbapenem resistance in Acinetobacter baumannii. Antimicrob. Agents Chemother. 49: 3198-3202.

Li, J., Nation, R.L., Owen, R.J., Wong, S., Spelman D, Franklin C. Antibiograms of multidrug-resistant clinical Acinetobacter baumannii: promising therapeutic options for treatment of infection with colistin resistant strains. Clin. Infect. Dis., 45: 594-8.

Manchanda, V., Sanchaita, S., Singh, N.P. 2010. Multidrug resistant Acinetobacter. J. Glob. Infect. Dis., 2(3): 291-304.

Montefour, K., Frieden, J., Hurst, S., Helmich, C., Headley, D. et al. 2008. Acinetobacter baumanni emerging multidrug resistant pathogen in critical care. Crit Care Nurse, 28: 15-25.

Munoz-Price, L.S., Robert, A. Weinstein. 2008. Acinetobacter Infection:Current concepts. N. Engl. J. Med., 358: 12711281.

Muntasir, I., Omer, Samia, A., Gumaa, Abdullatif, A., Hassan, Khaled, H., Idris, Osama, A., Ali, Mustafa, M., Osman, Mahmmoud, S., Saleh, Nagla, A., Mohamed, Mustafa, M., Khaled, Sinha, Agarwal, S., Srivastava, M Singh Analysis of carbapenemresistant Acinetobacter from a tertiary care setting in North India; Indian J. Med. Microbiol., 31(1): 6063.
Namita, Jaggi, Pushpa Sissodia, Lalit Sharma. 2012. Acinetobacter baumannii isolates in a tertiary care hospital: Antimicrobial resistance and clinical significance, J. Microbiol. Inf. Dis., (2): 57-63 JMID.

Neetu Gupta, Nageswari Gandham, Savita Jadhav, Ravindra Nath Mishra. 2015. Isolation and identification of Acinetobacter species with special reference to antibiotic resistance. $J$. Natural Sci. Biol. Med., Vol 6.Issue 1: 159-162.

Neonakis, L.K., Spandidos, D.A., Petinaki, E. 2010. Confronting multidrugresistant Acinetobacter baumannii: a review. Int. J. Antimicrob. Agents, 102-9.

Poonam Sharma, Yousuf, U.L. Bashir, Sarjiwan Kaur, Parmeet Kaur, Aruna Aggarwal. 2015. Emerging antimicrobial resistance and clinical relevance of Acinetobacter isolates in a tertiary care hospital of rural area of Punjab, India; J. Microbiol. Antimicrobial Agents, (1): 8-12.

Patwardhan, R.B., P.K. Dhakephalkar, K.B. Niphadka, B.A. Chopade, 2008. A study on nosocomial pathogens in ICU with special reference to multiresistant Acinetobacter baumannii harbouring multiple plasmids, Indian J. Med. Res., pp 178-187.

Rahul Kamble, et al. August 2012 Acinetobacter species in Health Care setting: Clinical significance and Antimicrobial sensitivity Int. J. Curr. Microbiol. App. Sci., 4(4): 861-869

Rasmussen, B.A., Bush, K. 1997. Carbapenem-hydrolyzing $\quad \beta$ lactamases. Antimicrob Agents Chemother., 41: 223-232.

Rit, K., Saha, R. 2012. Multidrug-resistant Acinetobacter infection and their susceptibility patterns in a tertiary care 
hospital. Nigerian Med. J., 53(3): 126128.

Rosenbaum, P., Aureden, K., Cloughessy, M., Goss, L., Kassai, M., Streed, S.A. 2010. Association for Professionals in Infection Control and Epidemiology. Washington, DC: 2010. Guide to the elimination of multidrug resistant Acinetobacter baumannii transmission in Healthcare Settings.

Schreckenberger, P.C., Daneshvar, M.I., Weyant, R.S., Hollis, D.G. 2007. Acinetobacter, Achromobacter, Chryseobacterium, Moraxella, and other non fermentative gram negative rods. In: Murray PR, Baron EJ, Jorgensen $\mathrm{JH}$, Landry ML, Pfaller MA, eds. Manual of clinical microbiology. 9th ed. Washington, DC: ASM Press, 770802.

Shanthi, M., Sekar, U. 2009. Multi-drug resistant Pseudomonas aeruginosa and Acinetobacter baumannii infections among hospitalized patients: risk factors and outcomes. J. Assoc. Physicians India, (11):687693. [PubMed]

Sinha, M., Srinivasa, H., Macaden, R. 2007. Antibiotic resistance profile \& extended spectrum beta-lactamase (ESBL) production in Acinetobacter species. Indian J. Med. Res., 126(1): 63-7.

Walther-Rasmussen, J., Hoiby, N. 2006. OXA-type carbapenemases. $J$. Antimicrob. Chemother., 57: 373383.

\section{How to cite this article:}

Harekrishna Nath and Dipa Barkataki. 2016. Prevalence of ESBL and MBL producing Acinetobacter Isolates in Clinical Specimens in Tertiary Care Hospital, Assam, India. Int.J.Curr.Microbiol.App.Sci. 5(11): 515-522. doi: http://dx.doi.org/10.20546/ijcmas.2016.511.060 